\title{
Analysis of the potential disadvantages of chemical countermeasures against the transfer of radiocaesium to plants
}

\author{
J. Guillén ${ }^{1}$, A. Baeza ${ }^{2}$ and N. Mocanu ${ }^{3}$ \\ ${ }^{1}$ LARUEX, Dept. of Applied Physics, C.U.M., University of Extremadura, c/ Sta. \\ Teresa de Jornet 38, 06080 Mérida (Badajoz), Spain \\ ${ }^{2}$ LARUEX, Dept. of Applied Physics, Faculty of Veterinary Science, \\ University of Extremadura, Avda. de la Universidad s/n, 10071 Cáceres, Spain \\ ${ }^{3}$ Dept. of Physics of Life and Environment, Horia Hulubei National \\ Institute of Research and Development for Physics and Nuclear Engineering, \\ Box MG-6, 077125 Mägurele-Bucharest, Romania \\ e-mail: fguillen@unex.es
}

\begin{abstract}
Countermeasures to reduce the transfer of a radionuclide from soil to plants are usually based on saturating the soil with a stable element that is a chemical analogue of that radionuclide. But when a radioactive contamination event occurs, it is seldom due to the evacuation of a pure radionuclide such as ${ }^{137} \mathrm{Cs}$. Instead, a mixture of radionuclides may be released into the environment. The countermeasures applied to reduce the transfer of one of them may also influence other radionuclides. We have developed an experimental procedure to check whether radiocaesium countermeasures are appropriate when other radionuclides are also involved, or have some disadvantages that may limit their use. It consists of growing wheat seeds under controlled laboratory conditions using Neubauer's plantlet method. This method has been in use for many years as a small-scale screening procedure to check a soil's capacity to provide the potassium that plants need as a nutrient. In particular, we used it to study the effectiveness and the disadvantages of the application of a potassium salt fertilizer to a soil contaminated with ${ }^{134} \mathrm{Cs},{ }^{90} \mathrm{Sr}$, and ${ }^{60} \mathrm{Co}$. This countermeasure indeed reduced the uptake of ${ }^{134} \mathrm{Cs}$, but it also enhanced the uptake of ${ }^{60} \mathrm{Co}$. This enhancement was higher for the wheat stems than for the roots. For ${ }^{90} \mathrm{Sr}$, there was observed a slight increase due to the fertilizer.
\end{abstract}

\section{INTRODUCTION}

The use of countermeasures to avoid transfer of radionuclides from soil to plants is usually based on the saturation of the soil with a stable element chemically analogue to a certain radionuclide. Because of its greater proportion, the stable element is supposed to be taken up to a greater degree than its radioactive counterpart [1]. One of the most usual countermeasures for radiocaesium is the application of chemicals rich in potassium. But when a radioactive contamination event occurs, it is frequently not due to the evacuation of a pure radionuclide, such as ${ }^{137} \mathrm{Cs}$. Instead, a mixture of radionuclides is released into the environment. However, there is little information available about the effect that radiocaesium countermeasures may have on the transfer of other radionuclides to plants.

We have developed an experimental protocol to check whether the radiocaesium countermeasures are also adequate for other radionuclides or have some disadvantage that may limit their use. The protocol consists of growing wheat seeds under controlled laboratory conditions using Neubauer's plantlet method [2]. This method is based on the finding that the soil has a limited amount of naturally mobile forms of potassium to be transferred to plants. Thus, the plants will take up mostly or completely the readily available forms of potassium in the soil, and the rate of this uptake will be faster the greater their density in the cultivated area or the lower the initial supply level of the nutrient. Then, 
if no supplementary potassium is added as fertilizer to the soil, the plants may extract part of its less available forms or some available chemical analogues to cover their nutritional needs. The method has been used for many years as a small-scale screening procedure to check a soil's capacity to provide the necessary potassium for plants as nutrient. In the present work, we apply the method to study the effectiveness and the disadvantages of the application of a potassium salt to a soil contaminated with ${ }^{137} \mathrm{Cs},{ }^{90} \mathrm{Sr}$, and ${ }^{60} \mathrm{Co}$.

\section{MATERIAL AND METHODS}

\subsection{Experimental Protocol}

$850 \mathrm{~g}$ of dry soil, previously passed through a steel sieve (mesh size of $2 \mathrm{~mm}$ ), was contaminated in the laboratory with a solution containing a mixture of ${ }^{134} \mathrm{Cs},{ }^{60} \mathrm{Co}$, and ${ }^{90} \mathrm{Sr}$. The specific activities of the radionuclides applied to the soil were 3200, 30600 , and $690 \mathrm{~Bq} / \mathrm{kg} \mathrm{d.w.,} \mathrm{respectively.} \mathrm{Following}$ the addition of the radionuclides, the soil was mixed manually to obtain a uniform distribution of the radionuclides in its mass. This homogeneity of the radioactive contamination of the soil was checked by measuring the activity of ${ }^{134} \mathrm{Cs}$ and ${ }^{60} \mathrm{Co}$ in 6 samples of the soil, taken at random from the whole amount. The dispersion of the measured values was $3.3 \%$ for ${ }^{134} \mathrm{Cs}$ and $2.2 \%$ for ${ }^{60} \mathrm{Co}$.

We then used this soil in an experimental laboratory model of a simple soil-plant system based on a slight modification of the procedure originally described in NEUBAUER's plantlet method [2]. This involved growing wheat plantlets to the "two-leaves" stage on a limited amount of soil, from the caryopsis (seed) phase, in a period of time of approximately three weeks. The trials were carried out in triplicate in PVC pots containing $50 \mathrm{~g}$ of soil and 100 selected wheat caryopsides with germination viability of over $90 \%$. Prior to sowing the seeds, all the pots were moistened to just $10 \%$ below the total water capacity of $36 \%$, weighed, and then sealed hermetically with transparent PVC foil (to avoid water loss by evaporation) and left for three weeks for the soil to reach ionic equilibrium. After that period, the wheat caryopsides were distributed over the soil of each pot, which was then again covered with transparent PVC foil for the first week to prevent excessive water loss by evaporation. Three weeks later, the wheat plantlets had reached the "two-leaves" stage, and were harvested, separating in each replicate the harvested stems and roots. The samples were kept under controlled laboratory conditions, with no direct exposure to sunlight, at room temperature, and with the soil moisture adjusted periodically to the level of the initial moisture of the soil.

To evaluate the influence of chemical countermeasures, in some experiments we added a potassiumbased fertilizer (in the form of $\mathrm{KCl}$ ) to the soil at the standard rate of $200 \mathrm{~kg} \mathrm{~K} / \mathrm{ha}$, which implied in our case the addition of $100 \mathrm{mg} \mathrm{K} /$ pot. There were thus four experiments:

$>$ Exp. A: Wheat plantlets grown in uncontaminated and unfertilized soil.

- Exp. C: Wheat plantlets grown in radioactively contaminated, unfertilized soil.

- Exp. AF: Wheat plantlets grown in uncontaminated, potassium-fertilized soil.

$>$ Exp. F: Wheat plantlets grown in radioactively contaminated, potassium-fertilized soil.

Experiments AF and A, in which wheat plantlets were grown with and without added potassium-based fertilizer in uncontaminated soil, acted as control experiments to check the plantlet method.

\subsection{Radioactivity analyses}

Gamma spectrometry was carried out using an n-type germanium detector with a $25 \%$ relative efficiency, a $1.87 \mathrm{keV}$ resolution for the $1332 \mathrm{keV}{ }^{60} \mathrm{Co}$ peak, and a peak-to-Compton ratio of $57.5: 1$.

The procedure followed for strontium extraction was based on separation by ion exchange columns [3]. First, the sample was calcined at $600^{\circ} \mathrm{C}$ to eliminate the organic matter prior to the radiochemical separation. Then it was acid digested, ethylenediaminetetraacetic acid (EDTA) was added to chelate the calcium present, and the $\mathrm{pH}$ was adjusted to 4.8 . The sample was passed through a Dowex $50 \mathrm{~W} \times 8$ 
resin column which only retains strontium at that $\mathrm{pH}$. The strontium was recovered from the resin with $\mathrm{NaCl}$ and then precipitated at $\mathrm{pH} 8$ as $\mathrm{SrCO}_{3}$ onto a 5-cm diameter striated planchet. The chemical recovery was determined by gravimetry. Once ${ }^{90} \mathrm{Sr}-{ }^{90} \mathrm{Y}$ equilibrium had been reached, the sample was measured in a low background gas-flow proportional counter (Canberra Model 2401).

\section{RESULTS AND CONCLUSIONS}

The activity levels detected for ${ }^{134} \mathrm{Cs},{ }^{60} \mathrm{Co}$, and ${ }^{90} \mathrm{Sr}$, and the dry masses obtained in experiments $\mathrm{C}$ and $\mathrm{F}$, are listed in Table 1. The addition of the potassium-based fertilizers had little effect on the amount of plant mass obtained in experiment $F$ relative to $C$. There was observed a slight increase of the mass of the stem relative to the roots. However, the effect of this fertilizer on the activity level of ${ }^{134} \mathrm{Cs}$ was greater. The activity level in the stem was reduced from a mean value of $230 \mathrm{~Bq} / \mathrm{kg}$ d.w. to almost the detection limit ( $60 \mathrm{~Bq} / \mathrm{kg} \mathrm{d.w.})$, and indeed in only one replicate did we detect ${ }^{134} \mathrm{Cs}$ above that threshold, $(134 \pm 60) \mathrm{Bq} / \mathrm{kg}$ d.w. In the roots, there was only a slight decrease in the ${ }^{134} \mathrm{Cs}$ activity level which was not statistically significant. This decrease in the ${ }^{134} \mathrm{Cs}$ uptake by the stems was predictable, as it had been observed previously by other workers for different species of plants [1, 3-5].

However, the effect of the potassium fertilizer was not limited to reducing the radiocaesium uptake by the wheat plantlets. The activity levels of ${ }^{60} \mathrm{Co}$ showed that the addition of the potassium fertilizer enhanced the uptake of this radioisotope by both stem and roots. In addition, it was observed that the relative increase in the uptake of ${ }^{60} \mathrm{Co}$ was greater in the stems than in the roots, with factors of 4.6 and 2.2, respectively. This suggested that the addition of $\mathrm{KCl}$ as fertilizer, with the probable increase in $\mathrm{K}^{+}$in the soil solution, made the ${ }^{60} \mathrm{Co}$ content of the soil more available for uptake. Lastly, the activity levels for ${ }^{90} \mathrm{Sr}$ with the potassium fertilizer seemed to be slightly higher than those obtained in the unfertilized experiment, although, due to the associated uncertainties, the differences were not statistically significant.

Table 1. Mean values and standard deviations of the dry mass obtained in each experiment, expressed in $g$ d.w., and of the ${ }^{134} \mathrm{Cs},{ }^{60} \mathrm{Co}$, and ${ }^{90} \mathrm{Sr}$ activity levels, expressed in $\mathrm{Bq} / \mathrm{kg}$ d.w., detected in stems and roots obtained in experiments $\mathrm{C}$ (without added potassium-based fertilizer) and $\mathrm{F}$ (with added potassium-based fertilizer). S/R means the ratio Stem/Root. D.L. = Detection Limit.

\begin{tabular}{|c|c|c|c|c|c|}
\hline \multicolumn{2}{|c|}{ Experiment } & Mass (g d.w.) & ${ }^{134}$ Cs & ${ }^{\mathbf{6 0}} \mathbf{C o}$ & ${ }^{\mathbf{9 0}} \mathbf{S r}$ \\
\hline \multirow{3}{*}{$\mathrm{C}$} & Stem & $1.22 \pm 0.05$ & $230 \pm 60$ & $3140 \pm 460$ & $890 \pm 80$ \\
\cline { 2 - 6 } & Root & $0.573 \pm 0.015$ & $930 \pm 190$ & $32800 \pm 4000$ & $280 \pm 28$ \\
\cline { 2 - 6 } & S/R & $2.13 \pm 0.12$ & $0.25 \pm 0.08$ & $0.096 \pm 0.018$ & $3.2 \pm 0.6$ \\
\hline \multirow{3}{*}{$\mathrm{F}$} & Stem & $1.39 \pm 0.07$ & D.L. & $14400 \pm 2100$ & $1000 \pm 40$ \\
\cline { 2 - 6 } & Root & $0.58 \pm 0.06$ & $890 \pm 190$ & $71000 \pm 8700$ & $350 \pm 70$ \\
\cline { 2 - 6 } & S/R & $2.42 \pm 0.24$ & D.L. & $0.20 \pm 0.04$ & $3.0 \pm 0.7$ \\
\hline
\end{tabular}

The process of transfer of radionuclides from soil to the different fractions was quantified by means of the transfer factors, TF, as defined in Equation (1). The TF values for ${ }^{134} \mathrm{Cs}$, ${ }^{60} \mathrm{Co}$, and ${ }^{90} \mathrm{Sr}$, corresponding to stems and roots and are listed in Table 2.

$$
\mathrm{TF}=\frac{\mathrm{Bq} / \mathrm{kg} \mathrm{d} \cdot \mathrm{w} .(\text { stem } / \mathrm{root})}{\mathrm{Bq} / \mathrm{kg} \text { soil }}
$$

In the unfertilized experiment, $\mathrm{C}$, the highest transfer factor for the stems was that of ${ }^{90} \mathrm{Sr}$, much greater than those of ${ }^{60} \mathrm{Co}$ and ${ }^{134} \mathrm{Cs}$. But for the roots, the highest was ${ }^{60} \mathrm{Co}$, followed by ${ }^{90} \mathrm{Sr}$ and ${ }^{134} \mathrm{Cs}$. This suggested a different accumulation of radionuclides in stem and roots. The application of potassium fertilizer led to decreased TF values for ${ }^{134} \mathrm{Cs}$ in stems, within the range $<0.39-0.83$, whereas the TF values for root were of the same order as in the unfertilized experiment. ${ }^{134} \mathrm{Cs}$ had the lowest transfer factor in all the experiments, and was located mainly in the roots. The $\mathrm{TF}$ values for ${ }^{60} \mathrm{Co}$ increased 
significantly with the addition of the potassium fertilizer, although the pattern of distribution was not changed. ${ }^{60} \mathrm{Co}$ accumulated preferentially in roots in both experiments. The effect of the potassium fertilizer on ${ }^{90} \mathrm{Sr}$ was a slight increase of the mean TF values in stems and roots, but also with no change in the pattern. ${ }^{90} \mathrm{Sr}$ was accumulated mainly in the stems. The different patterns of the TF's for stem and roots are listed in Table 3.

Table 2. Mean values and standard deviations of the transfer factors of ${ }^{134} \mathrm{Cs},{ }^{60} \mathrm{Co}$, and ${ }^{90} \mathrm{Sr}$ detected in stems and roots obtained in experiments $\mathrm{C}$ (without added potassium-based fertilizer) and $\mathrm{F}$ (with added potassium-based fertilizer). D.L. $=$ Detection Limit.

\begin{tabular}{|c|c|c|c|c|}
\hline \multicolumn{2}{|c|}{ Experiment } & $\mathbf{T F}\left({ }^{\mathbf{1 3 4}} \mathbf{C s}\right)$ & $\mathbf{T F}\left({ }^{\mathbf{6 0}} \mathbf{C o}\right)$ & $\mathbf{T F}\left({ }^{\mathbf{9 0}} \mathbf{S r}\right)$ \\
\hline \multirow{2}{*}{$\mathrm{C}$} & Stem & $1.44 \pm 0.35$ & $2.0 \pm 0.3$ & $25.9 \pm 2.4$ \\
\cline { 2 - 5 } & Root & $5.8 \pm 1.2$ & $21.5 \pm 2.6$ & $8.2 \pm 0.8$ \\
\hline \multirow{2}{*}{$\mathrm{F}$} & Stem & D.L. & $9.4 \pm 1.4$ & $29.0 \pm 1.5$ \\
\cline { 2 - 5 } & Root & $5.6 \pm 1.2$ & $46.4 \pm 5.7$ & $10.1 \pm 2.0$ \\
\hline
\end{tabular}

Table 3. Patterns of transfer of ${ }^{134} \mathrm{Cs},{ }^{60} \mathrm{Co}$, and ${ }^{90} \mathrm{Sr}$ from soil to stems or roots of wheat plantlets.

\begin{tabular}{|c|c|c|}
\hline Fertilizer & Stem & Root \\
\hline None (Exp. C) & ${ }^{90} \mathrm{Sr}>{ }^{60} \mathrm{Co} \approx{ }^{134} \mathrm{Cs}$ & ${ }^{60} \mathrm{Co}>{ }^{90} \mathrm{Sr}>{ }^{134} \mathrm{Cs}$ \\
\hline $\mathrm{KCl}($ Exp. F) & ${ }^{90} \mathrm{Sr}>{ }^{60} \mathrm{Co} \gg{ }^{134} \mathrm{Cs}$ & ${ }^{60} \mathrm{Co}>{ }^{90} \mathrm{Sr}>{ }^{134} \mathrm{Cs}$ \\
\hline
\end{tabular}

The analysis of the effect of addition of potassium fertilizer on the uptake of radionuclides other than radiocaesium gave an unexpected result. Although its addition indeed reduced the uptake of ${ }^{134} \mathrm{Cs}$ by the wheat stems, it increased significantly, by a factor of 4.6 , that of ${ }^{60} \mathrm{Co}$ by the same plant fraction. It was not possible to observe this in natural ecosystems, in which the amount of ${ }^{60} \mathrm{Co}$ is negligible. However, it is a result to be taken into account in the case to apply chemical countermeasures like potassium fertilizers to soils contaminated by a mixture of manmade radionuclides.

\section{Acknowledgments}

We wish to thank the Junta de Extremadura for the research grant given in 2006 to Dr Nicolae Mocanu for the project entitled "Estudio de la transferencia efectiva entre suelo y planta" from which this study is a part.

\section{References}

[1] Nisbet A.F., Mocanu N. and Shaw S., Sci. Tot. Environ., 149 (3) (1994) 145-154.

[2] Mocanu N. and Breban D.C., J. Radioanal. Nucl. Chem., 249 (3) (2001) 633-637.

[3] Gascó C. and Álvarez A. Sp SIN 0081-3397, 617 CIEMAT, Madrid, 1998.

[4] Nisbet A.F., Konoplev A.V., Shaw G., Lembrechts J.F., Merckx R., Smolders E., Vandecasteele C.M., Lonsjog H., Carini F. and Burton O., Sci. Tot. Environ., 137 (1993) 173-182.

[5] Fesenko S.V., R.M. Alexakhin, M.I. Balonov, I.M. Bogdevitch, B.J. Howard, V.A. Kashparov and N.I. Sanzharova. An extended critical review of twenty years of countermeasures used in agriculture after the Chernobyl accident. Sci. Tot. Environ., 383 (1-3) (2007) 1-24. 